\title{
A Short Note on Our Study on Gestational Diabetes Mellitus from Bedside to Bench
}

\author{
Jie Yan and Huixia Yang* \\ Department of Obstetrics and Gynecology, Peking University First Hospital, Beijing, China
}

Gestational Diabetes Mellitus (GDM) is a common complication during pregnancy. The initial diagnosis criteria were established almost 50 years ago and the debate on GDM diagnosis still persists in the global professional societies today.

In the 1960s, O'Sullivan and Mahan [1] firstly brought up the GDM diagnostic criteria by measuring the blood glucose levels in 752 pregnant women based on 3-hour $100 \mathrm{~g}$ oral glucose tolerance test (OGTT). They further validated the criteria by comparing future chances of developing Type 2 diabetes in mothers in a larger cohort ( $29 \%$ in abnormal OGTT vs. $17 \%$ in normal OGTT). It provided the evidence that the importance of detecting GDM during pregnancy. In 1979, the U.S. National Diabetes Data Group (NDDG) converted the blood glucose values proposed by $\mathrm{O}^{\prime}$ Sullivan to the equivalent values measured in plasma and recommended the following diagnostic criteria for GDM using 3-hour $100 \mathrm{~g}$ OGTT: a fasting plasma glucose $\geq 5.8 \mathrm{mmol} / \mathrm{L}$ (105 mg/dL); a 1-hour result of $\geq 10.6 \mathrm{mmol} / \mathrm{L}(190 \mathrm{mg} /$ $\mathrm{dL})$; a 2 -hour result of $\geq 9.2 \mathrm{mmol} / \mathrm{L}(165 \mathrm{mg} / \mathrm{dL})$ and a 3-hour result of $\geq 8.1 \mathrm{mmol} / \mathrm{L}(145 \mathrm{mg} / \mathrm{dL})[2]$.

The American Diabetes Association (ADA) and many other medical groups worldwide also accept the NDDG recommendation [3]. But different groups may set variable cutoff values to detect glucose abnormalities in pregnancy. The variability between different criteria largely came form the difficulties in converting glucose levels provided by $\mathrm{O}^{\prime}$ Sullivan to the equivalent values measured by modern techniques in plasma [4].

In China, most health care facilities and professionals adopted NDDG criteria to diagnose GDM. Nevertheless, one flaw showed up rather soon after applying the criteria in Chinese population. Most pregnant women could not stand for the glucose load and they appeared nausea and vomiting after taking $100 \mathrm{~g}$ glucose. Meanwhile, evidence from China showed $100 \mathrm{~g}$ OGTT response curve was similar to $75 \mathrm{~g}$ OGTT pattern in non-pregnant individuals. Thus, we switched to 3-hour $75 \mathrm{~g}$ OGTT and continued to use NDDG cutoff values to diagnose GDM. We were still lacking the data during pregnancies to validate these criteria. In 1993, Dong et al. proposed the new GDM diagnostic criteria in China after analyzing 75 g OGTT data obtained from 350 healthy pregnant women in Peking University First Hospital: a fasting plasma glucose $\geq 5.5 \mathrm{mmol} / \mathrm{L}(100 \mathrm{mg} / \mathrm{dL})$; a 1 -hour result of $\geq 10.2 \mathrm{mmol} / \mathrm{L}(185 \mathrm{mg} / \mathrm{dL})$; a 2 -hour result of $\geq 8.2 \mathrm{mmol} / \mathrm{L}(148 \mathrm{mg} /$ $\mathrm{dL})$ and a 3-hour result of $\geq 6.6 \mathrm{mmol} / \mathrm{L}(120 \mathrm{mg} / \mathrm{dL})$ [5]. Although, the new criteria did not gain wide acceptance due to the limited participants, Dong's study reminded us that $75 \mathrm{~g}$ OGTT values seemed much lower compared to the $100 \mathrm{~g}$ OGTT values in pregnant women. Then it was not appropriate to borrow NDDG criteria to diagnose GDM using $75 \mathrm{~g}$ glucose load. Otherwise, we underestimated the incidence of GDM in China and detected less GDM than it should be.

On the other hand, ADA started to recommend two criteria testing with $100 \mathrm{~g}$ or $75 \mathrm{~g}$ OGTT since the Fourth International WorkshopConference on Gestational Diabetes Mellitus [6]. The second criteria reduced the glucose load to $75 \mathrm{~g}$ and maintained the same cut points at fasting, 1-hour and 2-hour. In 1980 report, World Health Organization
(WHO) included GDM as a type of diabetes and recommended directly adopting the same diagnostic criteria as for non-pregnant individuals based on a $75 \mathrm{~g}$ OGTT [7].

They were not evidence-based practice. Universal strategy for GDM diagnosis was needed worldwide. The major challenge of new GDM diagnostic criteria was aiming to identify the pregnancies with increased risk for adverse perinatal outcomes. The Hyperglycemia Adverse Pregnancy Outcome (HAPO) study, an international multicenter study of a cohort of 25,505 pregnant women tested with 2-hour $75 \mathrm{~g}$ OGTT, formulated the GDM diagnostic criteria based on a 75 g OGTT [8]. In 2008, the International Association of Diabetes and Pregnancy Study Groups (IADPSG) reviewed results obtained from HAPO study and examined associations of maternal glycemia levels and perinatal and long-term outcomes in offspring. The IADPSG Consensus Panel recommended the following diagnostic criteria for GDM when at least one cut point was reached or exceeded: a fasting plasma glucose $\geq 5.1 \mathrm{mmol} / \mathrm{L}(92 \mathrm{mg} / \mathrm{dL})$; a 1 -hour result of $\geq 10.0$ $\mathrm{mmol} / \mathrm{L}(180 \mathrm{mg} / \mathrm{dL})$; and a 2 -hour result of $\geq 8.5 \mathrm{mmol} / \mathrm{L}(153 \mathrm{mg} /$ dL) [9].

After reviewing the data, the Ministry of Health $(\mathrm{MOH})$ of China accepted IADPSG criteria and published the uniform diagnostic criteria for GDM in China on 1 July 2011 [10]. It has been reported by our group the incidence of GDM in China is as high as $17.5 \%$ after applying the new criteria [11].

Nevertheless, there is no consensus on GDM diagnostic criteria at an early stage depending on the level of fasting plasma glucose (FPG). In order to determine the role of FPG in GDM diagnosis at the first prenatal visit, we initiated a study recruiting 17,186 pregnant women in 13 hospitals in China [11]. We found pregnant women with FPG between 6.1 and 7.0 should be diagnosed and treated as GDM; women with FPG between 5.1 and 6.1 should be provided nutrition and exercise advice and OGTT should also be performed at 24-28 weeks to diagnose GDM by then [11]. We also recommended measuring FPG only to screen GDM at 24-28 weeks in low-resource regions [12].

Meanwhile, we pay a lot of attention to reveal the underlying molecular mechanism of GDM as well as the consequences of offspring influenced by intrauterine hyperglycemia exposure. The concept of developmental origins of health and disease $(\mathrm{DOHaD})$ raised the

*Corresponding author: Huixia Yang, Xi'anmen Street No.1, Peking University First Hospital, Beijing, China, Tel: +86 13601165721; Fax: +86 66551211; E-mail: yanghuixia@bjmu.edu.cn

Received December 16, 2013; Accepted January 20, 2014; Published January 27,2014

Citation: Yan J, Yang H (2014) A Short Note on Our Study on Gestational Diabetes Mellitus from Bedside to Bench. J Mol Genet Med S1: 014. doi: 10.4172/1747 0862.S1-014

Copyright: (c) 2014 Yan J, et al. This is an open-access article distributed under the terms of the Creative Commons Attribution License, which permits unrestricted use, distribution, and reproduction in any medium, provided the original author and source are credited 
hypothesis that metabolic diseases may be influenced by adverse intrauterine environment during a sensitive period and emphasize the importance of balanced nutrition in utero. There are several established animal models to study the consequences of offspring exposed to intrauterine hyperglycemia. Streptozotocin (STZ) is a chemical widely used to establish diabetic animal models by specifically destroying $\beta$ cells of the pancreas in mammals [13]. Our further morphological study of pancreases confirmed the smaller pancreatic islets in diabetic mothers induced by STZ [14].

A single large dose of STZ (50 mg/kg) was administered intra peritoneally on the 5th day of gestation to induce intrauterine severe hyperglycemia model [15]. Low birth weight offspring can usually be obtained from this model. However, the high glucose levels of diabetic patients or severe GDM women have been mostly well controlled during the pregnancy. It is more realistic to establish a GDM model with intrauterine mild or moderate hyperglycemia exposure. Furthermore, the new criteria diagnosed more GDM including women previously thought to be normal for pregnancy. Thus, in order to determine the effects of intrauterine mild hyperglycemia, we induced the rat model with high birth weight offspring by a single low dose of STZ $(25 \mathrm{mg} / \mathrm{kg})$ [14]. Here, we will summarize the main observations and findings in the established rat models with different maternal glucose levels.

\section{The Consequences of Offspring Exposed to Intrauterine Severe Hyperglycemia in Rat Model}

The birth weight of offspring obtained from severely diabetic mothers was significantly lower than the controls. The early catchup growth rate was found in offspring exposed to intrauterine severe hyperglycemia and they maintained the similar body weight as controls after 9 weeks [16]. Besides, our study also showed overfeeding in early life could decrease insulin sensitivity in the offspring of diabetic mothers measured by hyper insulinaemiceu glycaemic clamp.

The offspring of severely diabetic mothers displayed normal blood glucose level and elevated insulin level indicating decreased insulin sensitivity in the offspring. However, further morphological study of pancreases of offspring showed no change in pancreatic islets [16]. The altered expression of genes involved in gluconeogenesis in liver may contribute to the insulin resistance in offspring born form diabetic mothers [16].

The offspring of diabetic mothers also showed higher levels of triglyceride and cholesterol at 26 weeks [16]. We also found mean arterial pressure was increased at 26 weeks by exposure to intrauterine severe hyperglycemia in offspring [16].

The brain injury was reported in offspring of diabetic mothers and the abnormal fetal brain development was associated with the increase of maternal serum levels of advanced glycation end-products (AGE) as well as the enhancement expression of receptor for advanced glycation end-products (RAGE) in fetal brain tissues [17]. AGE is a complex group of compounds that have been implicated in diabetes related complications. We suggest AGE and RAGE may play an important role in fetal brain injury obtained from diabetic mothers and micronutrients may ameliorate the brain damage [17].

\section{The Consequences of Offspring Exposed to Intrauterine Mild Hyperglycemia in Rat Model}

The birth weight of offspring obtained from mildly diabetic mothers was significantly higher than the controls. It is no difference of body weight in adulthood for male offspring, whereas female offspring of mildly diabetic mothers revealed obese after 16 weeks [14].

The offspring of mildly diabetic mothers display normal blood glucose level at birth. Impaired glucose tolerance in adulthood was displayed under glucose tolerance test using glucose $(2 \mathrm{~g} / \mathrm{kg}$ body weight) given by gavage [14]. The offspring of diabetic mothers also showed higher level of triglyceride at 28 weeks [18]. We also found mean arterial pressure was increased from 12 weeks by exposure to intrauterine mild hyperglycemia in male offspring [14].

Overall, either early or long-term complications of offspring are influenced by maternal diabetes. It is indicated that certain developmental pattern has been programmed in utero. Epigenetics provides a potential explanation for different susceptibilities to metabolic disease after exposing various intrauterine environmental factors. The epigenetic mechanism will enhance our understanding by which intrauterine environmental factors can regulate gene expression and may take effect in the long term. DNA methylation and histone modifications are the two major epigenetic modifications. Intrauterine nutritional status and environmental factors may contribute to DNA methylation changes that may be "memorized" during the whole life and influence the gene expression in a later life. Future work is needed to answer this question.

\section{References}

1. O'Sullivan JB, Mahan CM (1964) Criteria for the oral glucose tolerance test in pregnancy. Diabetes 13: 278-285.

2. (1979) Classification and diagnosis of diabetes mellitus and other categories of glucose intolerance. National Diabetes Data Group. Diabetes 28: 1039-1057.

3. (1980) American Diabetes Association Workshop-Conference on gestational diabetes: summary and recommendations. Diabetes Care 3: 499-501.

4. Carpenter MW, Coustan DR (1982) Criteria for screening tests for gestational diabetes. Am J Obstet Gynecol 144: 768-773.

5. Dong ZG (1993) Oral glucose tolerance test in late pregnancy women. Zhonghua Fu Chan Ke Za Zhi 28: 136-138, 187.

6. Metzger BE, Coustan DR (1998) Summary and recommendations of the Fourth International Workshop-Conference on Gestational Diabetes Mellitus. The Organizing Committee. Diabetes Care 21 Suppl 2: B161-167.

7. No authors listed (1980) WHO Expert Committee on Diabetes Mellitus: second report. World Health Organ Tech Rep Ser 646: 1-80.

8. HAPO Study Cooperative Research Group, Metzger BE, Lowe LP, Dyer AR Trimble ER, et al. (2008) Hyperglycemia and adverse pregnancy outcomes. N Engl J Med 358: 1991-2002.

9. Metzger BE, Gabbe SG, Persson B, Buchanan TA, Catalano PA, et al. (2010) International association of diabetes and pregnancy study groups recommendations on the diagnosis and classification of hyperglycemia in pregnancy. Diabetes care 33: 676-682.

10. Yang HX (2012) Diagnostic criteria for gestational diabetes mellitus (WS 331 2011). Chin Med J (Engl) 125: 1212-1213

11. Zhu WW, Yang HX, Wei YM, Yan J, Wang ZL, et al. (2013) Evaluation of the value of fasting plasma glucose in the first prenatal visit to diagnose gestational diabetes mellitus in china. Diabetes Care 36: 586-590.

12. Zhu WW, Fan L, Yang HX, Kong LY, Su SP, et al. ( 2013) Fasting plasma glucose at 24-28 weeks to screen for gestational diabetes mellitus: new evidence from China. Diabetes care 36: 2038-2040.

13. Rossini AA, Like AA, Chick WL, Appel MC, Cahill GF Jr (1977) Studies of streptozotocin-induced insulitis and diabetes. Proc Natl Acad Sci U S A 74 2485-2489.

14. Li X, Luo SJ, Zhang K, Yang HX (2012) Streptozotocin-induced maternal intrauterine hyperglycemia environment and its influence on development and metabolic in adult offspring with high birth weight in rats. Zhonghua Fu Chan Ke Za Zhi 47: 769-776. 
Citation: Yan J, Yang H (2014) A Short Note on Our Study on Gestational Diabetes Mellitus from Bedside to Bench. J Mol Genet Med S1: 014. doi: 10.4172/1747-0862.S1-014

Page 3 of 3

15. Liu HH, Zhang YL, Yang HX (2009) Intrauterine hyperglycemia and leptin resistance of offsprings.. Zhonghua Fu Chan Ke Za Zhi 44: 841-845

16. Ma JM, Zeng CJ, Zhang L, Shou C, Yang HX (2012) Increased hepatic peroxisome proliferator-activated receptor coactivator-1alpha expression precedes the development of insulin resistance in offspring of rats from severe hyperglycemic mothers. Chin Med J125: 1224-1229.
17. Luo SJ, Yang HX (2012) Roles of advanced glycation end products and its receptor on the fetal brain injury in pregnant rats with gestational diabetes mellitus. Zhonghua Fu Chan Ke Za Zhi 47: 364-367.

18. Zhang K, Li X, Yang HX (2013) Long-term effects of mild hyperglycemia exposure in utero and postnatal high fat diet on body weight and lipid metabolism in rat offsprings. Zhonghua Fu Chan Ke Za Zhi 48: 618-623. 